\title{
Delayed Tension Pneumothorax Detected 4 Days after Central Venous Catheterization - A case report -
}

${ }^{1}$ Department of Anesthesiology and Pain Medicine, and ${ }^{2}$ Institute of Health Sciences, Gyeongsang National University College of Medicine, Jinju, Korea

Seung Hwa Lee, M.D. ${ }^{1}$, Jae-Wan Lee, M.D. ${ }^{1}$, Ju-Tae Sohn, M.D. ${ }^{1,2}$, Hyo Min Lee, M.D. ${ }^{1}$, II-Woo Shin, M.D. ${ }^{1}$, Heon-Keun Lee, M.D. ${ }^{1}$, and Young-Kyun Chung, M.D. ${ }^{1}$

Pneumothorax is one of the most frequent complications of percutaneous central venous catheterization. Most significant pneumothoraces are easily detected on postcatheterization chest radiograph. However, we report a rare case of delayed tension pneumothorax detected 4 days after unsuccessful central venous catheterization via the infraclavicular subclavian vein, although initial postcatheterization and postoperative supine chest radiographs showed no active lesion. (Korean J Anesthesiol 2008; 54: S 59 61)

Key Words: central venous catheterization, delayed tension pneumothorax, pneumothorax.

More than $15 \%$ of patients who undergo central venous catheterization experience complications such as pneumothorax, arterial puncture, hematoma, venous thrombosis and catheter-related infection. ${ }^{1)}$ The most common complication of subclavian venous catheterization is pneumothorax, which can be partial, total, or under tension and accounts for $30 \%$ of all complications. ${ }^{2)}$ Most significant pneumothoraces are easily detected on postcatheterization chest radiograph. Here, we report a rare case of delayed tension pneumothorax detected 4 days after unsuccessful central venous catheterization via the infraclavicular subclavian vein.

\section{CASE REPORT}

A 24-year-old man was scheduled for suboccipital craniectomy in the prone position to remove astrocytoma at the cerebellar vermis and right cerebellar hemisphere. After induction of general anesthesia, catheterization of the right subclavian

Received : August 28, 2007

Corresponding to : Ju-Tae Sohn, Department of Anesthesiology and Pain Medicine, and Institute of Health Sciences, Gyeongsang National University College of Medicine, Chiram-dong, Jinju 660-702, Korea. Tel: 82-55-750-8586, Fax: 82-55-750-8142, E-mail: jtsohn@ nongae. gsnu.ac.kr vein was attempted four times unsuccessfully, and left subclavian vein catheterization was attempted twice unsuccessfully. Three anesthesiologists attempted central venous catheterization via the subclavian vein or left internal jugular vein. After two anesthesiologists failed to achieve central venous catheterization via the subclavian veins, a third anesthesiologist successfully placed a 5-French central venous catheter (BD Careflow ${ }^{\mathrm{TM}}$, Singapore) into the left internal jugular vein. We did not attempt central venous catheterization via internal jugular vein initially because neurosurgeon was concerned about increased intracranial pressure due to impaired internal jugular venous drainage. We did not find any aspirated air during any of the attempts for central venous catheterization. The postcatheterization supine chest radiograph showed no active lesion (Fig. 1). Anesthesia and the subsequent 5-hour operation were uneventful. The patient's peak inspiratory pressure during operation was $18-21 \mathrm{mmHg}$, and the supine chest radiograph taken immediately after the operation showed no active lesion (Fig. 2). The patient received mechanical ventilation in an effort to control intracranial pressure in the intensive care unit for 4 hours after the operation. The patient showed $18-20 \mathrm{mmHg}$ peak airway pressure and 98-100\% oxygen saturation during a 2-hour period of controlled mechanical ventilation (tidal volume: $450 \mathrm{ml}$, inspired oxygen fraction: 0.4 , respiratory rate: 


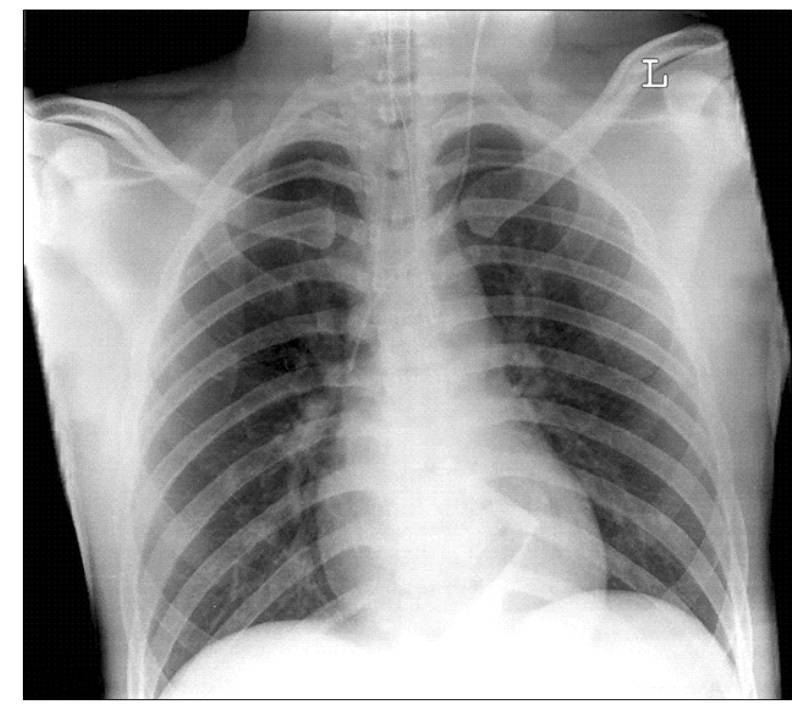

Fig. 1. Postcatheterization supine chest radiograph shows no active lesion.

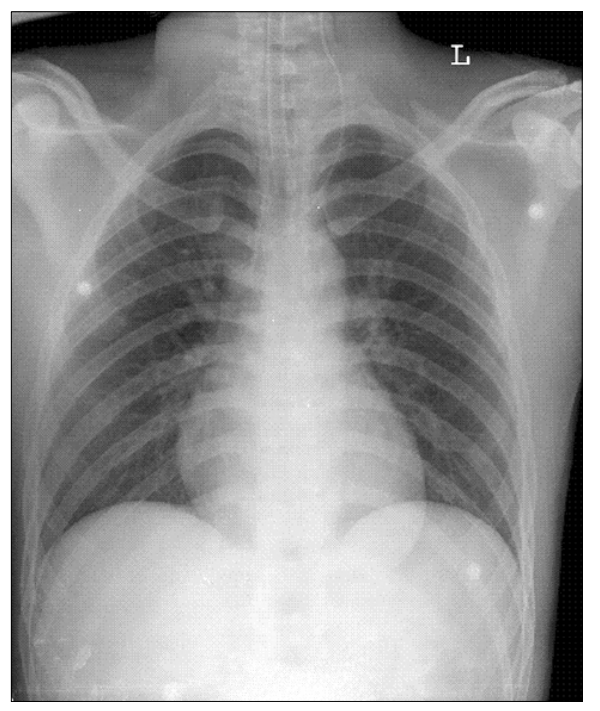

Fig. 2. Supine chest radiograph taken immediately after operation shows no active lesion.

$16 / \mathrm{min}$ ) in the intensive care unit. Finally, patient received 5 $\mathrm{cm} \mathrm{H}_{2} \mathrm{O}$ continuous positive airway pressure (inspired oxygen fraction: 0.4). Arterial blood gas analysis performed $30 \mathrm{~min}$ after the endotracheal tube was removed and showed the following results: $\mathrm{pH}, 7.36$; oxygen tension, $77 \mathrm{mmHg}$; carbon dioxide tension, $33 \mathrm{mmHg}$; and oxygen saturation, 95\%. The patient's peak airway pressure was $18-21 \mathrm{mmHg}$ in the prone position during operation and $18-20 \mathrm{mmHg}$ in the supine position during mechanical ventilation. There was no remarkable

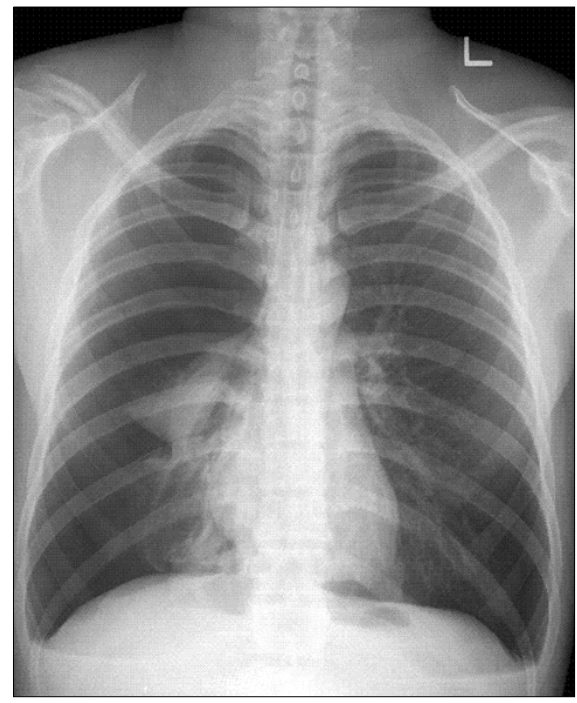

Fig. 3. Upright chest radiograph taken on post-operative day 4 due to patient complaints of chest discomfort and dyspnea shows pneumothorax on both sides (whole right lung: tension pneumothorax, left lung: pneumothorax).

difference in peak airway pressure between the prone and supine position during mechanical ventilation. The endotracheal tube and left internal jugular vein catheter were removed on post-operative day 1 . The patient's condition was stable until post-operative day 4, when he complained of chest discomfort and dyspnea. An upright chest radiograph taken at that time showed pneumothorax on both sides (whole right lung: tension pneumothorax, left lung: pneumothorax affecting about 20\% of the left lung) (Fig. 3). Arterial blood gas analysis showed severe hypoxemia ( $\mathrm{pH}$ : 7.39; arterial carbon dioxide tension: 37 $\mathrm{mmHg}$; arterial oxygen tension: $44 \mathrm{mmHg}$; bicarbonate: 21 $\mathrm{mmol} / \mathrm{L}$ ). The pneumothorax was successfully treated with right and left thoracotomy tubes (20-French) for 4 days.

\section{DISCUSSION}

A delayed pneumothorax, presumably caused by a slow pleural air leak, is a poorly recognized complication that can occur hours or days after central venous catheterization. ${ }^{3,4)}$ The overall reported incidence ${ }^{3,5)}$ of delayed pneumothorax is 0.4 to $0.6 \%$ of all central venous access attempts. Pneumothorax is much more common following a subclavian rather than a jugular approach, and generally associated with difficult or multiple approaches. ${ }^{5)}$ To exclude the presence of asymptomatic pneumothorax, a chest radiograph should be obtained within 4 hours after central venous catheterization of the subclavian and 
internal jugular veins. ${ }^{6}$ In all patients with a central venous catheter, an undetected delayed pneumothorax not visible on an early chest radiograph may occcur. ${ }^{7)}$ Patients with central venous catheters who have undergone positive pressure breathing should be examined carefully because they are at risk of developing a tension pneumothorax. ${ }^{7}$ We hypothesized that positive pressure ventilatory support in the intensive care unit might contribute to a large-sized tension pneumothorax that would not be visible on the initial chest radiograph. We thought that the severe hypoxemia observed in our patient on post-operative day 4 might have been associated with exhausted compensation mechanism and pneumothorax of the left lung. An upright expiratory chest radiograph is the procedure of choice for the evaluation of small pneumothorax, compared with lateral decubitus radiograph. ${ }^{8}$ In addition, in order to prevent pneumothorax and other relevant complications of central venous catheterization, it is advisable to use ultrasound guidance whenever possible. ${ }^{6}$ ) Depending on size and symptoms of pneumothorax, and particularly when a tension pneumothorax is suspected, treatment can vary from simple observation to chest tube insertion. ${ }^{6}$

In conclusion, if multiple attempts for central venous catheterization are made and the results of a postcatheterization chest radiograph appear to be normal but post-procedure pneumothorax is suspected in a patient who has undergone positive pressure ventilation after central venous catheterization, we recommend careful examination of the patient's vital signs and symptoms, daily upright expiratory chest radiograph, and extreme vigilance to detect delayed pneumothorax. If possible, the internal jugular vein approach is preferred over the subclavian vein approach.

\section{REFERENCES}

1. Taylor RW, Palagiri AV: Central venous catheterization. Crit Care Med 2007; 35: 1390-6.

2. Mitchell SE, Clark RA: Complications of central venous catheterization. AJR Am J Roentgenol 1979; 133: 467-76.

3. Spiliotis J, Kordossis T, Kalfarentzos F: The incidence of delayed pneumothorax as a complication of subclavian vein catheterisation. Br J Clin Pract 1992; 46: 171-2.

4. Choi YS, Lim OH, Yang MK, Chung IS, Kim YH: Delayed tension pneumothorax complicating subclavian vein catheterization and positive pressure ventilation. a case report. Korean $\mathrm{J}$ Anesthesiol 1998; 34: 660-4.

5. Plewa MC, Ledrick D, Sferra JJ: Delayed tension pneumothorax complicating central venous catheterization and positive pressure ventilation. Am J Emerg Med 1995; 13: 532-5.

6. Giacomini M, Iapichino G, Armani S, Cozzolino M, Brancaccio D, Gallieni M: How to avoid and manage a pneumothorax. J Vasc Access 2006; 7: 7-14.

7. Tan EC, van der Vliet JA: Delayed (tension) pneumothorax after placement of a central venous catheter. Ned Tijdschr Geneeskd 1999; 143: 1872-5.

8. Beres RA, Goodman LR: Pneumothorax: detection with upright versus decubitus radiography. Radiology 1993; 186: 19-22. 